\title{
Large Dispersive Interaction between a CMOS Double Quantum Dot and Microwave Photons
}

\author{
David J. Ibberson $\odot, 1,2,3,{ }^{*},+$ Theodor Lundberg $\odot,{ }^{4,2}$ James A. Haigh, ${ }^{2}$ Louis Hutin, ${ }^{5}$ Benoit Bertrand, ${ }^{5}$ \\ Sylvain Barraud, ${ }^{5}$ Chang-Min Lee, ${ }^{6}$ Nadia A. Stelmashenko $\odot,{ }^{6}$ Giovanni A. Oakes, ${ }^{4,2}$ \\ Laurence Cochrane $\odot,{ }^{7,2}$ Jason W.A. Robinson $\odot,{ }^{6}$ Maud Vinet, ${ }^{3}$ M. Fernando Gonzalez-Zalba $\odot,{ }^{2, \dagger, t}$ \\ and Lisa A. Ibberson $\oplus^{2}$ \\ ${ }^{1}$ Quantum Engineering Technology Labs, University of Bristol, Tyndall Avenue, Bristol BS8 1FD, United Kingdom \\ ${ }^{2}$ Hitachi Cambridge Laboratory, J.J. Thomson Avenue, Cambridge CB3 OHE, United Kingdom \\ ${ }^{3}$ Quantum Engineering Centre for Doctoral Training, University of Bristol, Tyndall Avenue, Bristol BS8 1FD, \\ United Kingdom \\ ${ }^{4}$ Cavendish Laboratory, University of Cambridge, J.J. Thomson Avenue, Cambridge CB3 0HE, United Kingdom \\ ${ }^{5}$ CEA/LETI-MINATEC, CEA-Grenoble, Grenoble 38000, France \\ ${ }^{6}$ Department of Materials Science \& Metallurgy, University of Cambridge, 27 Charles Babbage Road, Cambridge \\ CB3 OFS, United Kingdom \\ ${ }^{7}$ Nanoscience Centre, Department of Engineering, University of Cambridge, Cambridge CB3 OFF, \\ United Kingdom
}

(Received 5 April 2020; revised 18 December 2020; accepted 5 April 2021; published 5 May 2021)

\begin{abstract}
We report fast charge-state readout of a double quantum dot in a CMOS split-gate silicon nanowire transistor via the large dispersive interaction with microwave photons in a lumped-element resonator formed by hybrid integration with a superconducting inductor. We achieve a coupling rate $g_{0} /(2 \pi)=$ $204 \pm 2 \mathrm{MHz}$ by exploiting the large interdot gate lever arm of an asymmetric split-gate device, $\alpha=0.72$, and by inductively coupling to the resonator to increase its impedance, $Z_{r}=560 \Omega$. In the dispersive regime, the large coupling strength at the double quantum-dot hybridization point produces a frequency shift comparable to the resonator linewidth, the optimal setting for maximum state visibility. We exploit this regime to demonstrate rapid dispersive readout of the charge degree of freedom, with a SNR of 3.3 in $50 \mathrm{~ns}$. In the resonant regime, the fast charge decoherence rate precludes reaching the strong coupling regime, but we show a clear route to spin-photon circuit quantum electrodynamics using hybrid CMOS systems.
\end{abstract}

DOI: 10.1103/PRXQuantum.2.020315

\section{INTRODUCTION}

Electron spins in silicon quantum dots are promising candidates for large-scale quantum computation [1]. Their long coherence times enable high-fidelity single- and twoqubit gates [2-6] and their compatibility with industrial manufacturing processes [7-9] facilitates the fabrication of large quantum-dot arrays, and their integration with classical electronics [10]. All quantum-computing architectures

\footnotetext{
*david.ibberson@bristol.ac.uk

†mg507@cam.ac.uk

¥Current address: Quantum Motion Technologies, Windsor House, Cornwall Road, Harrogate HG1 2PW, United Kingdom

Published by the American Physical Society under the terms of the Creative Commons Attribution 4.0 International license. Further distribution of this work must maintain attribution to the author(s) and the published article's title, journal citation, and DOI.
}

aiming to achieve fault tolerance [11] require high-fidelity quantum-state readout. To implement quantum error correction (QEC), this readout must be achieved in timescales significantly shorter than the coherence time of the qubits $\left(T_{2}=28 \mathrm{~ms}\right.$ [12]), and ideally faster than the timescales of single- and two-qubit gates to avoid becoming the bottleneck in error-correction cycles. For the highest fidelity gates, these timescales are sub- $\mu \mathrm{s}$ [3] and $5 \mu \mathrm{s}$ [4], respectively. Renewed efforts have been directed at achieving this goal using charge sensors combined with amplification at the millikelvin stage [13] or with rf techniques [14,15], reaching a promising $97 \%$ fidelity in $1.5 \mu \mathrm{s}$ [16].

Circuit quantum electrodynamics (cQED) provides an alternative readout approach, which removes the necessity for a charge sensor and simplifies the qubit architecture [17-19]. The qubit interacts with photons in a detuned microwave cavity whose eigenfrequencies are modified conditional on the qubit state. Recent results have demonstrated this is a competitive approach for silicon spin qubits 
and have reached fidelities of $73 \%$ in $2.6 \mathrm{~ms}$ [20] and $82.9 \%$ in $300 \mu \mathrm{s}$ [21], when using hybrid resonant circuits, and $98 \%$ in $6 \mu \mathrm{s}$, when using on-chip resonators [22]. Although on-chip integration has enabled faster readout, it poses an important challenge for the future development of compact nanometre-scale spin qubit arrays, given that current microwave-resonator designs have characteristic length scales of the order of $100 \mu \mathrm{m}[23,24]$. Hybrid multichip modules give freedom to optimize the layout of the qubit layer without the spatial constraints of the microwave electronics and are likely to facilitate the development of two-dimensional qubit arrays [25,26], a requirement for the implementation of the surface code QEC [11]. However, the technology to achieve readout times compatible with QEC using hybrid multichip modules requires further development.

In this paper, we demonstrate fast charge-state readout of a CMOS double quantum dot (DQD) coupled to a high-impedance lumped-element resonator. We measure an interdot charge transition with a SNR of 3.3 in just $50 \mathrm{~ns}$. The signal, which represents that of a singlet outcome in singlet-triplet readout schemes, should enable high-fidelity spin readout in sub- $\mu$ s time scales. Our large signal is achieved by increasing the charge-photon coherent coupling rate to $g_{0} /(2 \pi)=204 \mathrm{MHz}$, comparable to those reported in fully integrated circuits [23,24]. The large coupling results directly from exploiting the large interdot gate lever arm of an asymmetric split-gate device, $\alpha=0.72$, and from inductively coupling to the resonator to increase its impedance. It allows us to operate the system in the intermediate dispersive regime, where the dispersive frequency shift of the microwave cavity, $\chi$, is as large as the photon decay rate, $\kappa$; the condition for maximum state visibility [27]. Furthermore, we show the scalability of the architecture by simultaneously reading two resonators, each of them containing a quantum-dot device, via frequency multiplexing. Finally, by tuning the charge configuration of the DQD, we reach the resonant regime of $\mathrm{CQED}$, where the qubit and resonator energies match. Although $g_{0} \gg \kappa$, the fast decay rate of the charge degree of freedom of the DQD precludes reaching the strong coupling limit in this experiment. By coupling to the longlived spin degree of freedom [28], it may be possible to reach the strong coupling limit, which has been achieved in $\mathrm{Si} / \mathrm{SiGe}$ heterostructures [23,29], but not yet in $\mathrm{Si} / \mathrm{SiO}_{2}$ systems. We note that the microwave circuitry presented here should readily improve the readout fidelity not only of CMOS-based devices but of any semiconductor-based system, especially those with a large gate lever arm such as topological qubits [30,31].

\section{ARCHITECTURE}

Our multimodule assembly consists of a silicon chiplet and a superconducting chiplet, connected via wire bonds.
The superconducting chiplet contains an elongated spiral inductor and a $50-\Omega$ microstrip waveguide, fabricated using optical lithography from an 80-nm-thick sputterdeposited $\mathrm{NbN}$ film on a sapphire substrate, shown in Fig. 1(a). The structure is designed to allow microwave radiation to be inductively coupled into the inductor from the waveguide. We connect the inner bond pad of the inductor to the $T 1$ gate of an $n$-type split-gate siliconon-insulator nanowire field-effect transistor (NWFET), see Fig. 1(b), and the outer bond pad to a bias tee to enable dc gating. More details of the circuit can be found in Appendix B.

The intrinsic-Si body of the nanowire, with a height of $7 \mathrm{~nm}$ and width $70 \mathrm{~nm}$, is covered by $6-\mathrm{nm} \mathrm{SiO}_{2}$ gate oxide and separated from the silicon substrate by a 145-nm buried oxide. Two gates, arranged face to face, wrap around the sides and part of the top surface of the nanowire. The gates have a length along the nanowire of $60 \mathrm{~nm}$, and surrounding the gates the nanowire is covered by $34-\mathrm{nm} \mathrm{Si}_{3} \mathrm{~N}_{4}$ spacers. The gap between the gates at the top of the nanowire is $40 \mathrm{~nm}$ wide, and is also covered by $\mathrm{Si}_{3} \mathrm{~N}_{4}$ [32]. An orange arrow on the top-down SEM image, Fig. 1(a), highlights the gap between the gates. We connect the inductor to the gate with the larger overlap to make use of the larger interdot gate lever arm, as we see later. Under the appropriate bias, a DQD forms within the corners of the NWFET, as illustrated in Fig. 1(b) [33]. We model a single valence electron in the DQD as a coupled two-level system with eigenfrequencies $\Omega_{ \pm}=$ $\pm(1 / 2) \Omega= \pm(1 / 2) \sqrt{\varepsilon^{2}+\left(2 t_{c}\right)^{2}}$, where $t_{c}$ is the tunnel coupling and $\varepsilon$ is the frequency detuning between dots.

The combined inductor-DQD system forms an $L C$ resonant circuit. We study the properties of the resonator by monitoring the amplitude and phase of the reflected signal when applying a microwave tone to the microwave input line, $\mathrm{MW}_{\text {in }}$, with the NWFET grounded at $12 \mathrm{mK}$, see Fig. 1(c). We find a resonance at $f_{0}=1.88 \mathrm{GHz}$, which can be fitted using a complex external $Q$ value [see red dashed line in Fig. 1(c)] [34,35]. From the fit and our experimentally measured inductance value $L=47 \mathrm{nH}$ [36], we deduce a total parasitic capacitance $C_{p}=150 \mathrm{fF}$, a characteristic impedance $Z_{r}=\sqrt{L / C_{p}}=560 \Omega$, an internal [external] decay rate $\kappa_{\text {int }} /(2 \pi)=1.31 \mathrm{MHz}\left[\kappa_{\text {ext }} /(2 \pi)=\right.$ $1.76 \mathrm{MHz}]$ and a total photon decay rate of $\kappa /(2 \pi)=3.07$ MHz. Moreover, we plot the phase shift $\phi$ across the resonance (blue trace) and observe a $2 \pi$ phase shift confirming that photons predominantly escape the resonator before decaying [37].

Next, at fixed frequency, we monitor the phase shift of the reflected signal $\phi$ as a function of the gate biases $V_{T 1}$ and $V_{B 1}$, see Fig. 1(d). An enhancement of the phase signal in a quasiperiodic honeycomb pattern is clearly visible. The lines correspond to charge bistable regions in a DQD where the charge susceptibility of the DQD is nonzero $[38,39]$. We infer the electron population in each quantum 
(a)

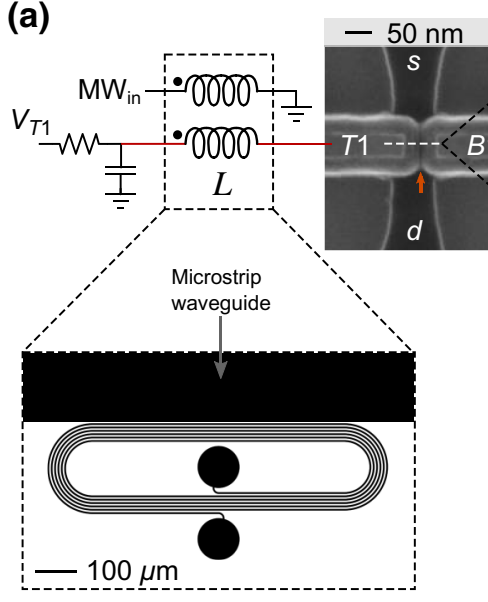

(b)

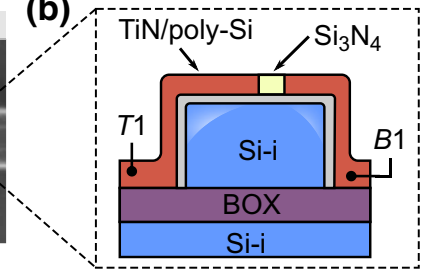

(c)

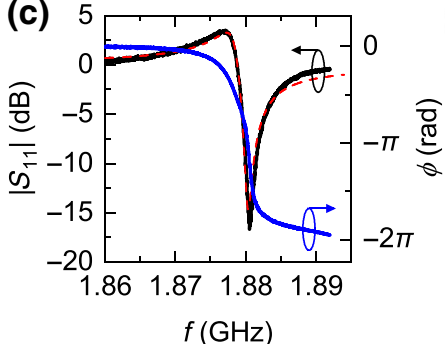

(d)

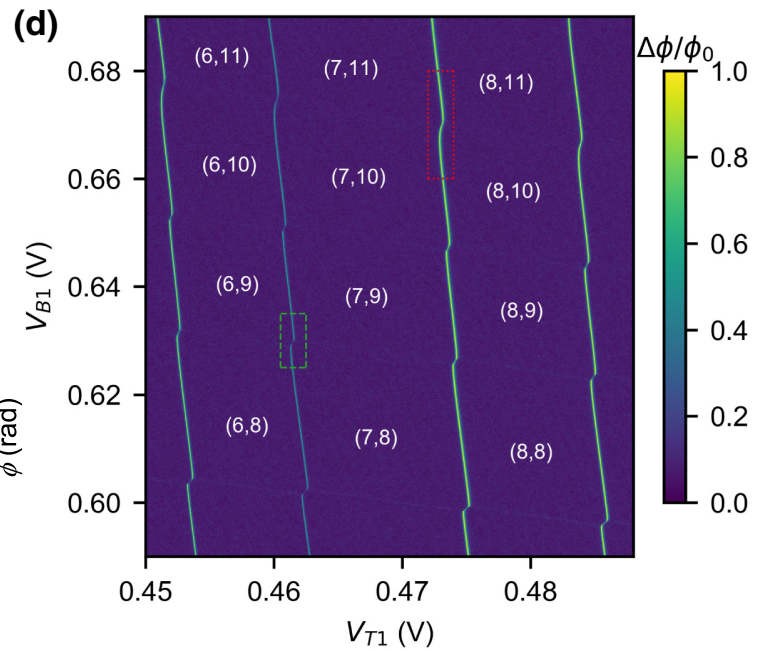

FIG. 1. Measurement setup, resonator geometry, and double quantum-dot characteristics. (a) Two mutually coupled inductors represent the inductive coupling between a microstrip waveguide and a spiral inductor with inductance $L$, the layout of which is shown below in the dashed box. Both elements are fabricated from the same $\mathrm{NbN}$ film on sapphire; the gap separating inductor and waveguide is $4 \mu \mathrm{m}$ wide. The inductor is wire bonded to a NWFET on a separate substrate (aluminium wire bonds are highlighted in red). The bias tee consists of a $100-\mathrm{pF}$ capacitor and a $100-\mathrm{k} \Omega$ resistor. A SEM image shows a top-down view of a similar split-gate NWFET, where the orange arrow highlights the insulating spacer between the two top gates and its misalignment from the center of the nanowire. (b) Cross-section schematic of the NWFET, cutting through the nanowire perpendicular to its length. Quantum dots are formed in the top corners of the intrinsic silicon body, indicated by white shading. (c) The phase (blue), amplitude (black), and fit (red) of a reflected microwave signal applied and measured at $\mathrm{MW}_{\text {in }}$ when the terminals of the NWFET are grounded. (d) The DQD charge stability diagram shown in normalized reflected phase data as $V_{T 1}$ and $V_{B 1}$ are varied. Numbers in parentheses indicate the electron numbers on each dot; the highlighted ICTs are measured in detail later.

dot by using a visible dot-to-reservoir transition of one dot as a charge sensor of the other dot, see Appendix D. Then we invert the role of the dots and count the number of electrons in the opposite dot [32]. The numbers in parentheses in Fig. 1(d) indicate the number of electrons on the dot under the $(T 1, B 1)$ gates. Interdot charge transitions (ICTs) at intersections of the horizontal and vertical lines are not visible for electron populations smaller than those presented on this plot, indicating insufficient wave-function overlap for tunneling to occur in the timescale of the resonator period [40].

In the remainder of this paper, we focus on studying the interaction between the resonator and the DQD. We use two ICTs with different tunnel couplings to access two different regimes. First, the dispersive regime, where the qubit frequency is greater than the resonator frequency, which we use to achieve fast readout of the charge state [red dotted box in Fig. 1(d)] and second, the resonant regime, where the qubit and resonator frequencies are equal [green dashed box in Fig. 1(d)].

\section{LARGE DISPERSIVE INTERACTION}

We bias the DQD to the ICT between charge states $(7,11)$ and $(8,10)$, see Fig. 2(a). The ICT and the $T 1$ dot-toreservoir (DTR) lines are visible, but the $B 1$ DTR ones are not because of the weak lever arm of the $T 1$ gate to the $B 1$ dot. We focus on the ICT and extract the interdot gate lever arm from the voltage shift of the transition as the magnetic field is increased, see Fig. 2(b). For an even-parity transition such as this, where the total number of valence electrons is even [for example a $(1,1) \leftrightarrow(2,0)$ transition], the position of the ICT shifts with a slope determined by $\alpha / g_{e} \mu_{B}$, where $g_{e}$ is the electron $g$-factor (approximately 2) and $\mu_{B}$ is the Bohr magneton [38]. The signal at $B>0 \mathrm{~T}$ originates from charge transitions between states with different spin numbers. The reason for spin transitions occurring under the microwave electric field is unknown, but could potentially be explained by electrically driven Landau-Zener transitions followed by a fast relaxation hotspot near the point of anticrossing [41]. We obtain $\alpha=0.724 \pm 0.006$. The large $\alpha$ is a direct consequence of the large asymmetric channel overlap of $T 1$ and represents the highest reported for any silicon device. Previous measurements of a device from the same wafer used the gate electrode with the smaller overlap $(B 1)$, which yielded $\alpha=0.345$ [32]. Achieving a large $\alpha$ is of great importance for $\mathrm{cQED}$ architectures since it relates directly to the dipole moment of the quantum system and hence to the coherent coupling constant $g_{0} /(2 \pi)=\alpha f_{0} \sqrt{Z_{r} / 2 R_{Q}}$, where $R_{Q}$ is the resistance quantum [42].

To explore the interaction between the resonator and the DQD, we measure the reflected spectrum of the resonator as we change the DQD frequency detuning, $\varepsilon$, via 

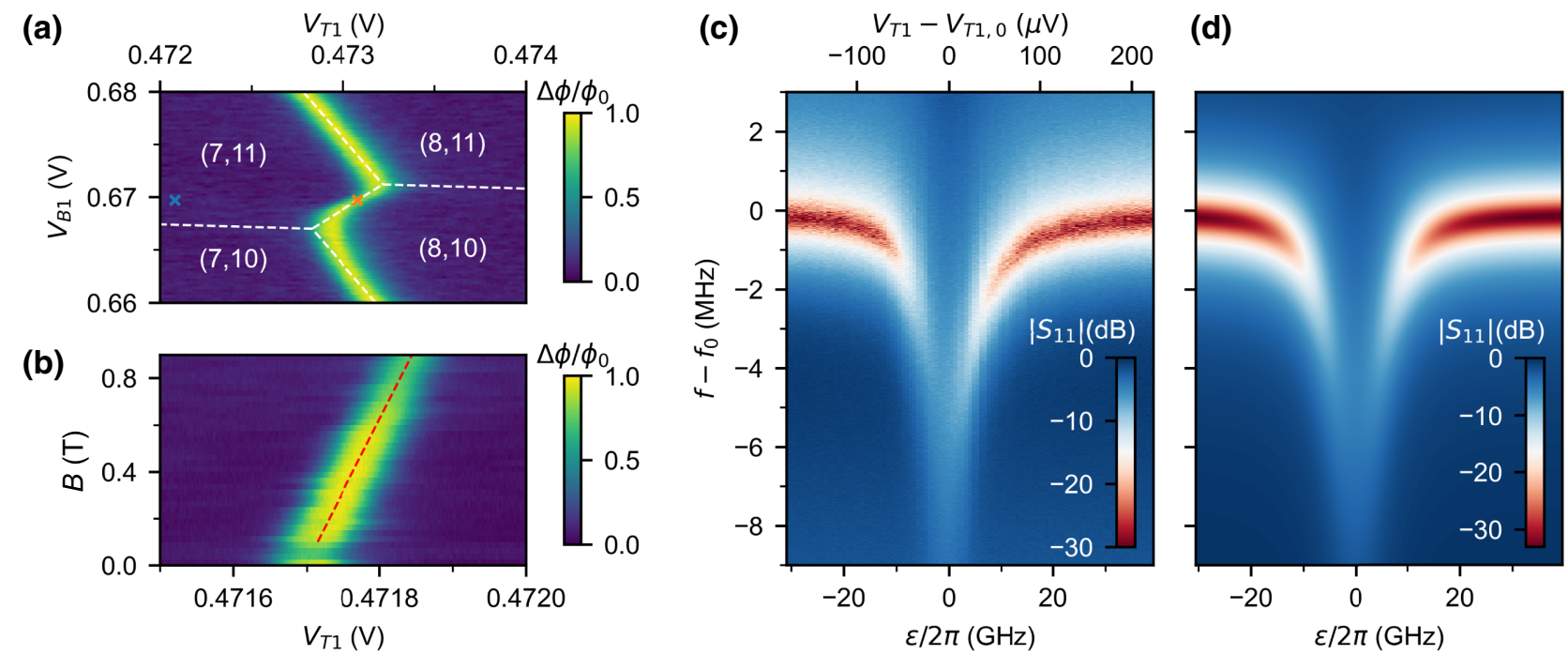

FIG. 2. Resonator-DQD interaction in the dispersive regime. (a) Reduced phase response of the resonator $\Delta \phi / \phi_{0}$ around the $(7,11) \longleftrightarrow(8,10)$ ICT. Color-coded crosses denote the positions of measurements on and off the ICT, respectively, see Fig. 3(a). (b) Magnetospectroscopy of the $(7,11) \longleftrightarrow(8,10)$ ICT, which we use to calculate the interdot gate lever arm. (c) Magnitude of the signal reflected from the cavity showing the shift in the resonant frequency as $V_{T 1}$ is swept across the ICT. (d) Simulated data, using Eq. (2), to reproduce the experimental data in (c).

$V_{T 1}$, see Fig. 2(c). We apply the input signal with a power of $-125 \mathrm{dBm}$ at $\mathrm{MW}_{\mathrm{in}}$, well below the onset of powerbroadening. At $\varepsilon=0$, the resonator exhibits a maximum frequency shift with respect to the bare resonator frequency, $\chi /(2 \pi)=4.1195 \pm 0.0006 \mathrm{MHz}$. This value is comparable to the bare cavity linewidth $\kappa /(2 \pi)$. In addition, we observe an increase in the effective resonator linewidth, $\kappa^{*}$, which we attribute to the interaction of the resonator with a fast decaying $\mathrm{DQD}$, as we confirm later.

To extract the relevant parameters of the resonator-DQD system, and to simulate the response, we model the DQD as a coupled two-level system, and the microwaves by a weak periodic field [43]. In the rotating-wave approximation, we obtain the effective Hamiltonian

$$
H_{\mathrm{tot}}=\hbar \Delta_{0} a^{\dagger} a+\frac{\hbar \Delta}{2} \hat{\sigma}_{z}+\hbar g_{\mathrm{eff}}\left(a \sigma_{+}+a^{\dagger} \sigma_{-}\right),
$$

where $\Delta_{0}=(2 \pi)\left(f_{0}-f\right), \quad \Delta=\Omega-(2 \pi) f_{0}, \quad g_{\text {eff }}=g_{0}$ $2 t_{c} / \Omega, \hat{\sigma}_{z}$ is the inversion operator, $\sigma_{+(-)}$are the raising and lowering operators of the DQD, and $a$ and $a^{\dagger}$ are the photon annihilation and creation operators. We use the input operator formalism and develop the HeisenbergLangevin equations of motion to obtain the reflection coefficient in the steady state when neglecting quantum-noise contributions

$$
\left|S_{11}\right|=\left|1+\frac{i \kappa_{\mathrm{ext}}}{\Delta_{0}-\frac{i \kappa}{2}+g_{\mathrm{eff}} \chi_{\mathrm{DQD}}}\right|^{2},
$$

where $\left|S_{11}\right|$ is the reflected power ratio, $\chi_{\mathrm{DQD}}=$ $g_{\text {eff }} /(-\Delta+i \gamma / 2)$ is the susceptibility of the DQD and $\gamma$ is the DQD decay rate. We use Eq. (2) to simulate the experimental results, see Fig. 2(d). From the $\varepsilon$-dependent shift in the resonance frequency, $\chi=$ $-g_{\text {eff }}^{2} \Delta /\left[\left(\Delta^{2}+\gamma^{2} / 4\right)\right]$, and the resonance linewidth increase $\kappa^{*}=\kappa+g_{\text {eff }}^{2} \gamma /\left(\Delta^{2}+\gamma^{2} / 4\right)$, see Appendix A, we determine the relevant system parameters: $g_{0} /(2 \pi)=$ $204 \pm 2 \mathrm{MHz}, 2 t_{c} /(2 \pi)=7.76 \pm 0.13 \mathrm{GHz}$ and $\gamma /(2 \pi)=$ $9.95 \pm 0.29 \mathrm{GHz}$. From these values, we can draw some important conclusions. Firstly, the DQD presents a fast decay rate, in line with reports in the literature for the charge degree of freedom in silicon MOS structures [44-46]. We discard Purcell-enhanced relaxation, $\gamma_{p} /(2 \pi)=\left(g_{0} / \Delta\right)^{2} \kappa /(2 \pi)=3.7 \mathrm{kHz}$ [47], and measurement-induced decoherence due to photon shot noise, $\gamma_{\phi} /(2 \pi)=(\sqrt{2 \bar{n}}-1) \kappa /(4 \pi)=25 \mathrm{MHz}$ [48] (where $\bar{n}$ is the average photon number) as the cause of the large $\gamma$, and instead attribute it to charge noise at the $\mathrm{Si} / \mathrm{SiO}_{2}$ interface. Secondly, although $\gamma \gg g_{0}$, we note that the coupling strength is much greater than the resonator linewidth (i.e., $\left.g_{0} \gg \kappa\right)$ and hence our system satisfies one of the requirements to reach the strong coupling regime. Finally, since $\Delta \gg g_{0}$ and $\chi / \kappa \approx 1$, our measurements are in the intermediate dispersive limit, relevant for high-fidelity qubit readout.

\section{DISPERSIVE READOUT PERFORMANCE}

We now evaluate the dispersive readout performance by measuring the SNR of the charge transition as a function of integration time, $t_{\text {int }}$. Using a continuous-wave excitation, we measure the $I$ and $Q$ demodulated components of the reflected signal at the two voltage positions marked 


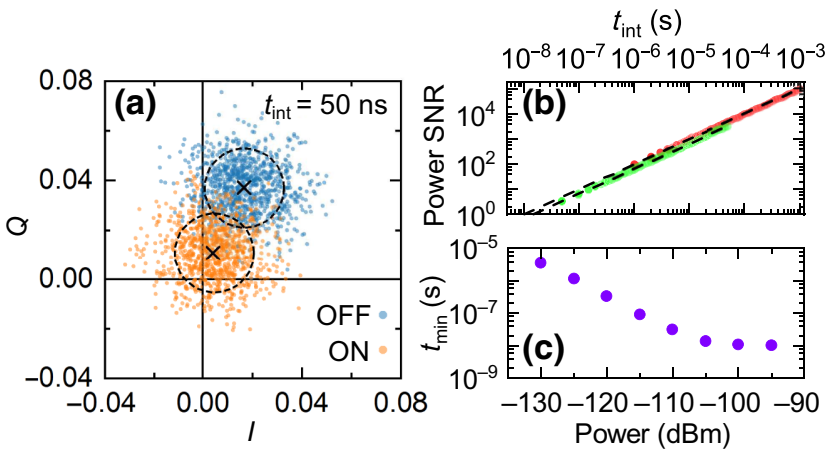

FIG. 3. Readout performance evaluation. (a) Distribution of the reflected signal in quadrature space, collected at the two points marked in Fig. 2(a) on and off the ICT signal. Each point is collected with an integration time $t_{\text {int }}=50 \mathrm{~ns}$. For each distribution, the black cross marks the mean and the dashed circle indicates the standard deviation of distances to the mean. (b) SNR dependence on $t_{\text {int }}$ for input power $=-100 \mathrm{dBm}$. Red data points are taken with a 1-MHz low-pass filter and the green data points are taken with a $20-\mathrm{MHz}$ low-pass filter. The dashed lines extrapolate the data to $\mathrm{SNR}=1$, from which we extract the minimum integration time, $t_{\min }$. (c) Decrease in $t_{\min }$ with increasing input power, which saturates due to power broadening at approximately $100 \mathrm{dBm}$ [49].

in Fig. 2(a): the Coulomb blockaded region (blue cross) and the charge degenerate regime (orange cross). This corresponds to the expected signal in a singlet-triplet spin readout scheme [20], in the limit where the valley splitting is larger than the tunnel coupling. Measurements of the valley splitting in excess of $500 \mu \mathrm{eV}$ have been reported for corner dots in the single-electron regime [28], which is much larger than the tunnel coupling measured in this work, $2 t_{c}=32 \mu \mathrm{eV}$. As shown in Fig. 3(a), the distributions of points at the two voltages are separated in the $I Q$ plane. We use this data to calculate the power SNR, given by $(A / B)^{2}$, where $A$ is the distance between the centers of the clusters (marked by black crosses), and $B$ is the average standard deviation of the two states (dotted circles). In Fig. 3(b), the red and green dots plot the SNR for two instrument configurations, using either a $1-\mathrm{MHz}$ or $20-\mathrm{MHz}$ maximum measurement bandwidth, respectively. From a linear fit to the data, we extract $t_{\min }$, defined as the integration time for which $\mathrm{SNR}=1$. At the optimal input power of $-100 \mathrm{dBm}$ [see Fig. 3(c)], for the $1-\mathrm{MHz}$ data we find $t_{\min }=10 \mathrm{~ns}$. This value is shorter than other silicon implementations, which have benefited from either a Josephson parametric amplifier (JPA) [50], or a fully integrated resonator [22]. Our result is comparable to the best reported in any dispersively detected semiconductor quantum dot, which was obtained in InAs and utilized a JPA [51]. Furthermore, following a simple readout fidelity model [52], we obtain a $99.7 \%$ electrical readout fidelity in $300 \mathrm{~ns}$. Using the $20 \mathrm{MHz}$ bandwidth configuration (green dots), we can experimentally access integration times down to $50 \mathrm{~ns}$. We note that the lower SNR in the $20-\mathrm{MHz}$ case is due to the comparably larger equivalent noise bandwidth of the filtering system. We measure a SNR of 3.3 at $t_{\text {int }}=50 \mathrm{~ns}$, for which the $I Q$ data is plotted in Fig. 3(a). This result agrees well with the estimated SNR in the intermediate dispersive regime SNR = $\left(\Delta^{2} h f_{0} k_{\text {ext }} t_{\text {int }}\right) /\left(8 \pi g_{0}^{2} k_{B} T_{N}\right)=2.6$, where $k_{B}$ is the Boltzmann constant and $T_{N} \approx 4 \mathrm{~K}$ is the noise temperature of the cryogenic amplifier, see Appendix F.

Finally, we highlight that this architecture can be easily extended to read out several qubits from a single transmission line by adding additional spirals along the length of the microstrip waveguide and employing frequencymultiplexing techniques $[53,54]$. We simultaneously read the charge states of two separate NWFETs embedded in resonators of different frequencies $(1.8$ and $2.4 \mathrm{GHz}$, corresponding to superconducting inductors with $L_{1}=47$ $\mathrm{nH}$ and $L_{2}=29 \mathrm{nH}$, respectively), see Appendix $\mathrm{C}$ for experimental details.

\section{RESONANT REGIME}

In our split-gate geometry, we are not able to tune the tunnel coupling independently, however $t_{c}$ can vary with electron occupation. We bias the DQD to a different ICT [highlighted by the green dashed rectangle in Fig. 1(d)] and investigate the effect of the DQD on the resonator in this case. In Fig. 4(a), we measure the resonator's frequency spectrum as we vary $\varepsilon$ across the $(6,9) \longleftrightarrow(7,8)$ transition, and observe a substantially different response with respect to the dispersive regime (also note the reduced span of the horizontal axis). In this case, close to $\varepsilon=0$, the resonator's frequency increases as opposed to the previous configuration. In addition,

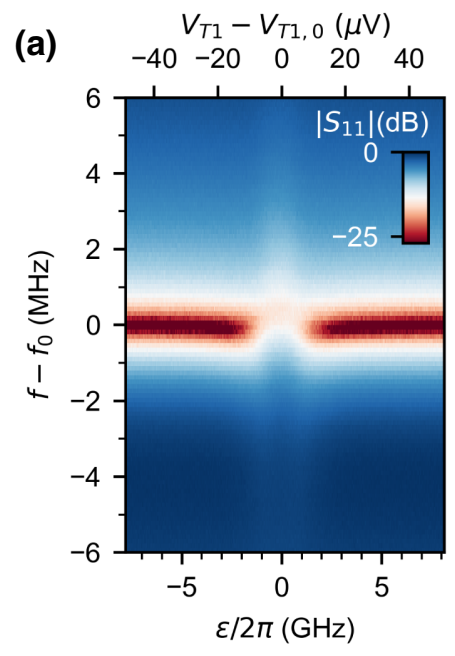

(b)

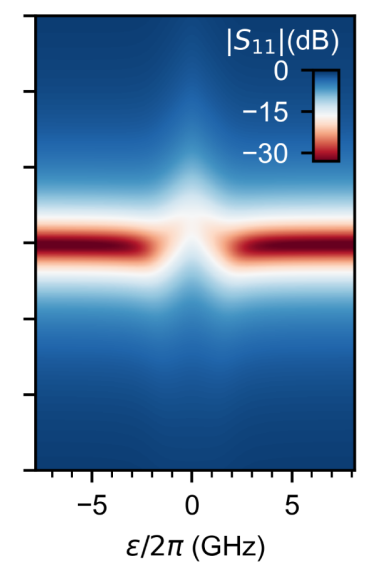

FIG. 4. Response of the resonator in the resonant regime. (a) Magnitude in $\mathrm{dB}$ of the power reflected at the resonator, as $V_{T 1}$ is varied across the $(6,9) \longleftrightarrow(7,8)$ ICT. (b) Simulation results based on Eq. (2). 
we again observe an increase of the effective resonator linewidth $\kappa^{*}$. We attribute these effects to the qubit and the resonator becoming resonant at some $\varepsilon$ where $\Omega=f_{0}$. However, due to the large DQD decay rate, we are unable to observe the vacuum Rabi-mode-splitting characteristic of the strong coupling regime. To confirm our hypothesis, we repeat the process described in Appendix A to fit the data. Using the lever-arm measurement $\alpha=0.66 \pm$ 0.01 , obtained from magnetospectroscopy of this ICT, we find a coupling strength $g_{0} /(2 \pi)=34.77 \pm 0.06 \mathrm{MHz}$, tunnel coupling $2 t_{c} /(2 \pi)=1065 \pm 2 \mathrm{MHz}$, and decay rate $\gamma /(2 \pi)=2.334 \pm 0.007 \mathrm{GHz}$. The smaller lever arm accounts for part of the reduction in $g_{0}$ compared with the dispersive regime, and a further reduction may be linked to the smaller tunnel coupling, analogous to the dependence of the coherent coupling rate on Josephson energy for superconducting qubits [55]. The reduced $\gamma$ in comparison to the dispersive regime can be understood by assuming phonon-mediated relaxation dominates, for which $\gamma \propto t_{c}^{3}$ [56]. Our simulations confirm that the system reaches the resonant regime since the tunnel coupling is lower than the frequency of the resonator. Furthermore, the simulation confirms that the fast decay rate of the DQD prevents us observing the strong coupling limit.

\section{CONCLUSION}

We present a multimodule-integrated circuit for fast readout of the charge state of quantum-dot devices enabled by a large charge-photon coupling rate. In our demonstration, the enhanced coupling results from two critical improvements. First, the large interdot gate lever arm, which arises from the thin gate oxide, the thin siliconon-insulator layer, and the asymmetric gate overlap of the nanowire. Second, the enhanced resonator impedance due to the reduced capacitance of the resonator, achieved by coupling inductively rather than capacitively $[36,37,50]$. In the dispersive regime, we achieve a frequency shift of the order of the resonator bandwidth, $\chi / \kappa \approx 1$, the condition for maximum state visibility. Furthermore, the measured coupling rate is greater than the resonator linewidth, satisfying one of the requirements for strong coupling. Future experiments aiming to achieve strong coupling to the charge degree of freedom in multimodule quantum systems should focus on decreasing the DQD charge decay rate. One strategy to do this is to move to high-mobility heterostructures where lower $\gamma$ have been demonstrated [57,58], possibly because of the reduced charge noise when compared to $\mathrm{Si} / \mathrm{SiO}_{2}$ interfaces. However, achieving a high $\alpha$ in those systems is a complex technical challenge because of the typically large separation between the gates and the quantum dot. Alternatively, coupling to the spin rather than the charge degree of freedom should provide an optimal route to cQED in hybrid superconductingCMOS systems, particularly if hole-based devices, which are subject to sizable spin-orbit coupling, are used $[59,60]$. Our work shows that, in the short term, it will be possible to reach the large coupling rates necessary for cQED without the necessity to integrate microwave resonators in a CMOS process. In the long-term, asymmetric gate designs made out of high kinetic inductance materials, like TiN, inductively coupled to a single input line, should provide a more scalable route to integrated CMOS cQED. Furthermore, if the large $\alpha$ of CMOS DQDs were combined with even higher impedance resonators, it will be possible to access the ultrastrong coupling regime [61]. We note that to achieve the fast readout presented in this paper, we did not use a JPA showing that there is room for further improvement. Finally, we expect that our approach should impact research aiming at improving the readout fidelity of Majorana-based quantum devices [30,31].

\section{ACKNOWLEDGMENTS}

This research has received funding from the European Union's Horizon 2020 Research and Innovation Programme under Grant Agreement No. 688539 [62]. M.F.G.Z. acknowledges support from the Royal Society and the Winton Programme of the Physics of Sustainability. D.J.I. is supported by the Bristol Quantum Engineering Centre for Doctoral Training, EPSRC Grant No. EP/L015730/1. T.L. and G.A.O. acknowledge support from EPSRC Cambridge NanoDTC EP/L015978/1 and L.C. acknowledges support from EPSRC Cambridge UP-CDT EP/L016567/1.

\section{APPENDIX A: $S$-PARAMETER ANALYSIS AND FITTING}

For the analysis of the frequency spectrum of the DQD resonator in Figs. 2 and 4 in the main paper, it becomes particularly useful to represent Eq. (2) in the form of a complex Lorentzian

$$
\left|S_{11}\right|=\left|1-\frac{i \kappa_{\mathrm{ext}}}{\omega-\omega_{0}+\frac{g_{\mathrm{eff}}^{2} \Delta}{\Delta^{2}+\gamma^{2} / 4}+\frac{i}{2}\left(\kappa+\frac{g_{\mathrm{eff}}^{2} \gamma}{\Delta^{2}+\gamma^{2} / 4}\right)}\right|^{2},
$$

where $\omega_{0}=(2 \pi) f_{0}$ is the resonant angular frequency of the resonator and the center frequency is given by

$$
\omega_{c}=\omega_{0}-\frac{g_{\mathrm{eff}}^{2} \Delta}{\Delta^{2}+\gamma^{2} / 4}
$$

and the effective linewidth of the resonator by

$$
\kappa^{*}=\kappa+\frac{g_{\mathrm{eff}}^{2} \gamma}{\Delta^{2}+\gamma^{2} / 4}
$$


In the limit $2 t_{c} \gg \gamma / 2$, Eq. (A2) presents the FWHM in $\varepsilon$ given by $\varepsilon_{1 / 2}=2 \sqrt{\left(2^{2 / 3}-1\right)} t_{c}$.

We now describe our method for extracting the coherent coupling rate $g_{0}$, tunnel coupling $t_{c}$, and charge relaxation rate $\gamma$ from the frequency spectroscopy data, see Fig. 2(c) for the dispersive regime, and Fig. 4(a) for the resonant regime. For each step in the detuning $\varepsilon$, we extract the resonance frequency $f_{c}$ and the FWHM of the resonance. In Fig. 5 we plot $f_{c}$ and the FWHM in (a) and (b), respectively, for the dispersive regime, and (c) and (d), respectively, for the resonant regime. We then fit these peak shapes using Eq. (A2) for $f_{c}$ and Eq. (A3) for the FWHM. For best accuracy, we fit simultaneously $f_{c}$ and FWHM with common parameters $g_{0}, t_{c}$, and $\gamma$. Fitting is performed using orthogonal distance regression (ODR), taking into account errors on both $x$ and $y$ axes for each plot. The dominant error on $\varepsilon$ stems from the uncertainty in $\alpha$, and the errors on $f_{c}$ and the FWHMs are given by the nonlinear least-squares fits used to extract their values from the $\left|S_{11}\right|$ data. The errors quoted on the fitted parameters, $g_{0}, t_{c}$, and $\gamma$, are obtained from the covariance matrix of the ODR fit. The quality of the resulting fits, indicated by the black dashed lines, gives high confidence in the calculated parameters.
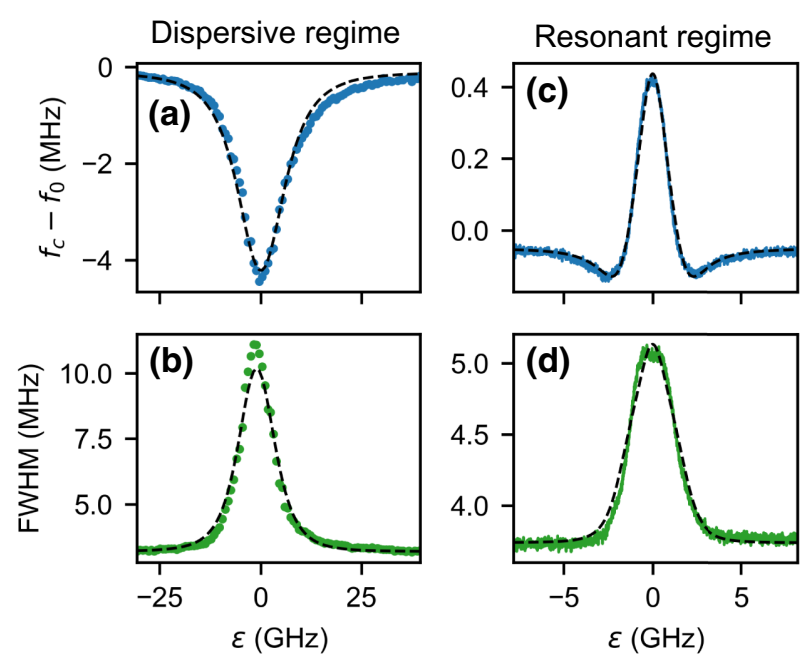

FIG. 5. Quality of model fitting. (a) Blue points plot the shift in the center frequency of the cavity, $f_{c}$, as the detuning is swept across the ICT in the dispersive regime. (b) Green points plot the change in FWHM of the cavity resonance across the ICT in the dispersive regime. For both (a),(b) the points are extracted from the data shown in Fig. 2(c), and the black dashed line represents the best-fit prediction from the model [see Fig. 2(d)]. (c),(d) Plot of the same quantities for the ICT in the resonant regime (see Fig. 4). For all panels, errors on $f_{c}$ and FWHM are omitted for clarity, and errors on $\varepsilon$ are too small to be visible. In (a),(b) the $f_{c}$ and FWHM error bars are too small to be visible, and for (c),(d), the errors are roughly the same magnitude as the random scatter in the points.

\section{APPENDIX B: RF CIRCUIT DESIGN AND OPERATION}

We present a photograph of the hybrid integrated system described in the main paper, showing the silicon NWFET and superconducting inductor on separate substrates, see Fig. 6. The $T 1$ gate of the silicon CMOS transistor is connected to the center of the $\mathrm{NbN}$ spiral inductor on the adjacent sapphire substrate, using an Al wire of length roughly $1500 \mu \mathrm{m}$ and diameter $17.5 \mu \mathrm{m}$. An on-PCB bias tee, not shown, is wire bonded to the outer bond pad of the spiral inductor, with an Al wire of similar length. The 50- $\Omega$ microstrip waveguide runs approximately vertically on the right of the figure. The insert magnifies the elongated sixturn spiral inductor, which is separated from the microstrip waveguide by a $4-\mu \mathrm{m}$ gap; the overall inductor footprint is $812 \times 326 \mu \mathrm{m}$, including bond pads.

The high impedance of the silicon NWFET split gate is impedance matched to the $50-\Omega$ microstrip waveguide, by embedding the device in an $L C$ resonator and designing the distance between the spiral and the waveguide to achieve critical inductive coupling. The bias-tee capacitor (NPO COG $100 \mathrm{pF}$ high $Q$ ) acts as a short circuit at the resonant frequency, thereby allowing high-quality factors to be achieved by isolating the bias-tee resistor.

We now present two models of the resonant system formed by the NWFET, PCB, superconducting chiplet, and bond wires, and attempt to reproduce the reflected spectrum data in Fig. 1(c). The first model consists of lumped elements only, illustrated in Fig. 7. In the second model, we use the Cadence ${ }^{\circledR}$ AWR $^{\circledR}$ AXIEM $^{\circledR}$ planar method-of-moments (MOM) software to simulate the $\mathrm{NbN} /$ sapphire/grounded-metal structure, which is then embedded in a lumped-element circuit representing the other structures that is simulated using Cadence ${ }^{\circledR}$ AWR $^{\circledR}$ Microwave Office ${ }^{\circledR}$, see Fig. 8.

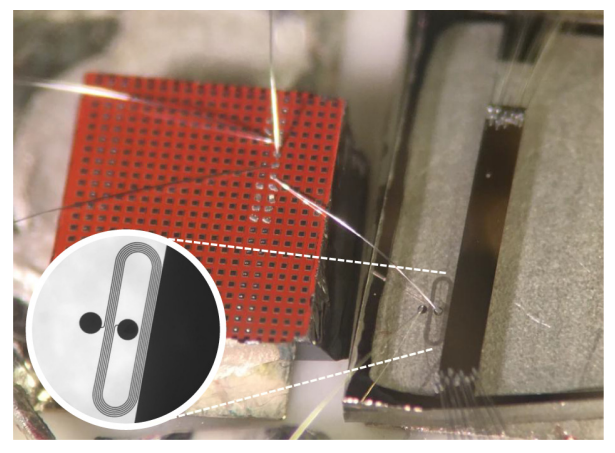

FIG. 6. Photograph of the multimodule setup. The silicon chip with an array of square bond pads is seen to the left, and to the right is the $\mathrm{NbN}$-on-sapphire substrate. The two modules are positioned adjacent to each other on a printed circuit board. The insert magnifies the elongated-spiral inductor adjacent to the superconducting $50 \Omega$ waveguide. 


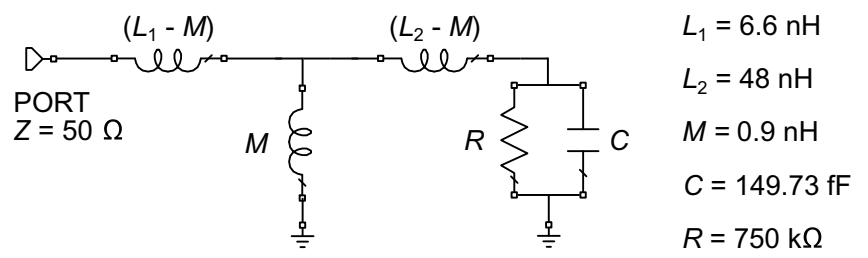

FIG. 7. Simplified circuit model. The inductively coupled spiral inductor and microstrip waveguide can be approximated as a $\mathrm{T}$ circuit. All of the parasitic capacitances in the system are combined with the gate capacitance into the one capacitor shown. The total losses are accounted for by the $750-\mathrm{k} \Omega$ resistor.

In the first model, the coupled inductances of the $\mathrm{NbN}$ waveguide and spiral are represented using a $\mathrm{T}$ circuit of inductors (see Fig. 7), where the central shunt inductance equals the mutual inductance, $M$, and the left and right inductors are the stray inductances of the waveguide and spiral, respectively, where $L_{1}$ is the inductance of the waveguide and $L_{2}$ is the spiral inductance. The capacitance, $C$, that completes the resonator includes the selfcapacitance of the spiral, the gate capacitance of the NWFET, and the total parasitic capacitances to ground. The resistor, $R$, accounts for internal losses, namely dielectric losses of the two substrates and resistance of nonsuperconducting metals in the bias tee. We find from MOM simulations of the sapphire chip that $M=0.9 \mathrm{nH}$, and deduce from our experimentally measured value of the spiral inductance (equal to $L_{2}-M$ ) that $L_{2}=48 \mathrm{nH}$. The remaining parameters are then obtained by fitting the model to the $\left|S_{11}\right|$ data in Fig. 1(c) with $M$ and $L_{2}$ fixed. The asymmetry of the data is accounted for by including an additional parameter in the fit function, specifying the angle of rotation in the complex plane about the point marked by an orange $X$ in Fig. 9(a). This rotation is caused

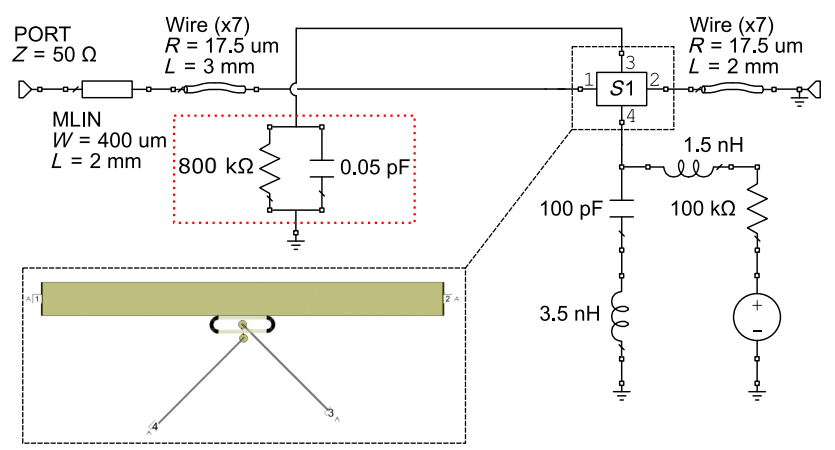

FIG. 8. AWR AXIEM EM simulations. We model the system, including parasitic contributions, using a three-dimensional planar MOM EM analysis simulator. The insert shows the model of the sapphire chip with the microstrip waveguide and spiral inductor. The NWFET is represented by a $800-\mathrm{k} \Omega$ resistor in parallel with a $50-\mathrm{fF}$ capacitor, highlighted by a red dotted box. The MLIN component resides on the Rogers 4003C PCB.
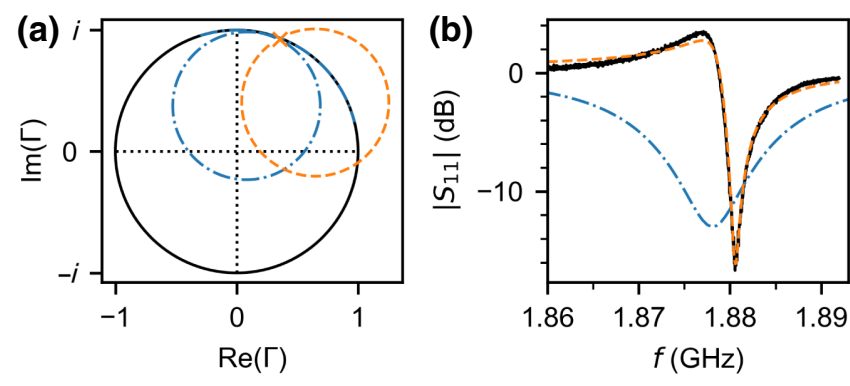

FIG. 9. Simulated resonator response. (a) Simulated reflection coefficient is plotted on a Smith chart for a range of frequencies around resonance. The orange dashed line gives the response simulated by the simplified circuit model shown in Fig. 7, which has rotated about its origin on the edge of the circle by $48^{\circ}$ to reproduce the asymmetry observed in the magnitude data. The blue dash-dot line gives the response simulated using the combination shown in Fig. 8. (b) We plot the absolute value of the reflection coefficient on the $\mathrm{dB}$ scale against frequency. The experimental data from Fig. 1(c) is included in black.

experimentally by interference of the radiation reflected from the resonator with radiation reflected at other boundaries, in this case most likely the grounded end of the waveguide $[63,64]$. Note that this model does not include the bias tee, making the assumption that it acts as a perfect ground in the vicinity of the resonant frequency.

In the second model, we construct a planar model of the sapphire chip including, from bottom to top, the grounded metal plane on top of the PCB, the sapphire of thickness $500 \mu \mathrm{m}$ and dielectric constant 11.58 , and the $\mathrm{NbN}$ microstrip and spiral. We model the $\mathrm{NbN}$ film as a perfect conductor with sheet inductance $1.35 \mathrm{pH} / \square$ to account for kinetic inductance, similar to other estimates [65]. This value is obtained by matching the simulated to the experimental resonant frequency of the unbonded spiral, which is measured at $3.04 \mathrm{GHz}$. Wire bonds are also modeled in the MOM simulation in order to connect the spiral to the off-chip elements. Connected to the outer bond pad via one of these wires is the bias tee, consisting of a 100-pF capacitor to ground, and a $100-\mathrm{k} \Omega$ resistor through which the $V_{T 1}$ bias is applied. In addition, two inductors of a few $\mathrm{nH}$ each are included to model PCB tracks and through-holes. Connected to the inner bond pad of the spiral, are a resistor and capacitor in parallel to model the NWFET, similar to the previous model. We find that, to match the experimental resonant frequency, this capacitance must be reduced to approximately $50 \mathrm{fF}$, indicating that the majority of the $150 \mathrm{fF}$ of the lumped-element model originates from the superconducting spiral. Moreover, separate simulations of the unbonded spiral also show that its capacitance is around $100 \mathrm{fF}$, which could be reduced in future experiments [66]. We investigate the effects of different lengths of the bond wires, which each contribute approximately 2 $\mathrm{nH}$ of inductance. For the bond wire between spiral and 
NWFET, varying its length $\pm 50 \%$ produces $<5 \%$ variation in the resonant frequency. A $50 \%$ variation in the length of the bond wire between the spiral and the bias tee shifts the resonant frequency by $<3 \%$. No significant changes to the resonance width or depth are observed.

The simulated reflection spectra of the two models are compared with the experimental data in Fig. 9(b). The lumped-element model shows excellent agreement with the data, whereas for the MOM simulation, while its resonant frequency is close to the experiment, the width is overestimated. This can be attributed to our choice of value for the resistor modeling internal losses, for which we use a value similar to the lumped-element model. This value is clearly an underestimate, from which we can conclude that the internal losses of the spiral predicted by the MOM simulation add a significant contribution. Note that varying the bias-tee capacitor and resistor values by their rated tolerances has no visible effect. The MOM simulation also does not reproduce the asymmetry of the data.

\section{APPENDIX C: FREQUENCY MULTIPLEXING}

We fabricate a second NbN-on-sapphire chip that included multiple inductors close to the microstrip waveguide for frequency-multiplexing experiments. Each inductor is wire bonded to separate bias tees and single-gate CMOS devices in the same configuration as before, enabling simultaneous measurement of two independent silicon quantum dots via the single microwave line. A 47-nH spiral inductor is bonded to a NWFET with gate length $(L) 50 \mathrm{~nm}$ and width $(W) 30 \mathrm{~nm}$ (device 1), and a 29-nH inductor is wire bonded to a NWFET with

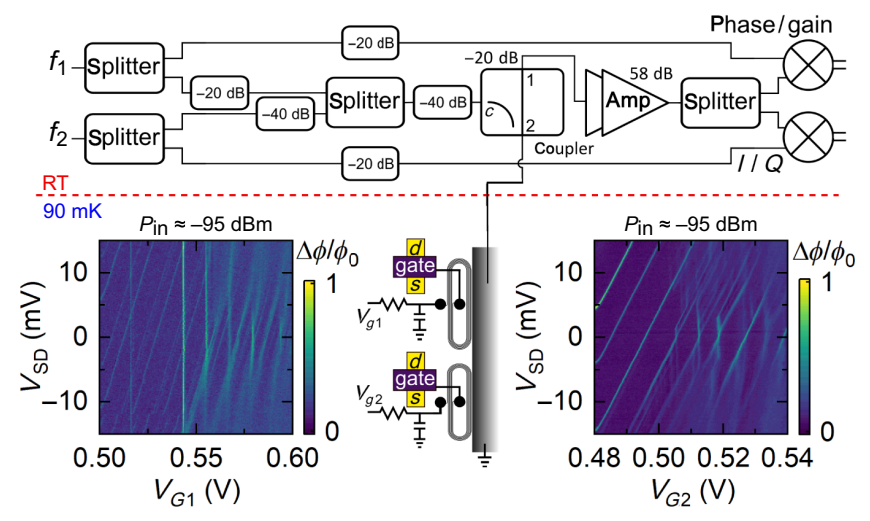

FIG. 10. Schematic of the frequency-multiplexing experiment and results. Tones from separate microwave sources $\left(f_{1}\right.$ and $\left.f_{2}\right)$ are combined and applied to two single-gate silicon NWFETs via a common microwave waveguide. The reflected signal is amplified at room temperature, divided, and then demodulated with the relevant reference signal $\left(f_{1}\right.$ is demodulated into phase and gain components using an Analog Devices AD8302, whereas $f_{2}$ is demodulated into $I$ and $Q$ using a Polyphase AD0540B). Coulomb diamonds for both devices are measured simultaneously at $1.78 \mathrm{GHz}$ (left plot) and $2.45 \mathrm{GHz}$ (right plot).

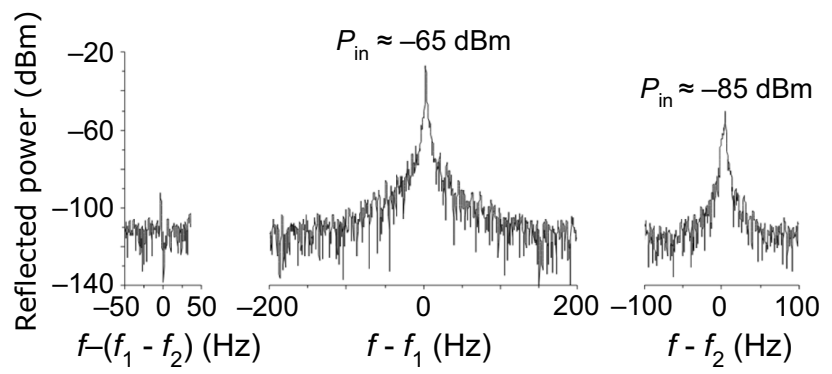

FIG. 11. Measurement of the intermodulation product, $f_{1}-f_{2}$, to characterise crosstalk. The intermodulation product is only observable in the multiplexing experiments when the input power of $f_{1}\left(f_{2}\right)$ is raised by approximately $30 \mathrm{~dB}(10 \mathrm{~dB})$ above the typical experimental level. The resulting product is at least $60 \mathrm{~dB}$ $(40 \mathrm{~dB})$ below the reflected $f_{1}\left(f_{2}\right)$ level.

dimensions $50 \mathrm{~nm}(L) \times 60 \mathrm{~nm}(W)$ (device 2$)$, giving resonant frequencies at $f_{1}=1.78 \mathrm{GHz}$ and $f_{2}=2.45 \mathrm{GHz}$, respectively. A two-tone probe signal is synthesized by combining $f_{1}$ and $f_{2}$ from individual microwave generators and applied to the microstrip waveguide via an unattenuated coaxial line in our dilution refrigerator, see Fig. 10. The reflected signal is amplified at room temperature, split into two channels, and then demodulated separately with the generated $f_{1}$ and $f_{2}$ tones. By manipulating the gate and bias voltages of the two NWFETs at the same time, we simultaneously acquired the two Coulomb diamond plots shown in Fig. 10. We quantify the crosstalk between the two measurements by measuring the signal power of the intermodulation product at $f_{1}-f_{2}$, see Fig. 11 . It is not possible to observe the intermodulation product above the noise floor when applying the same input powers used to acquire the Coulomb diamonds, however, increasing the power of the tones results in an observable intermodulation product, at least $60 \mathrm{~dB}(40 \mathrm{~dB})$ below the reflected $f_{1}\left(f_{2}\right)$ tones. The power of the intermodulation product is independent of the devices' gate voltages, indicating that there is no crosstalk at the resonator-device level.

Using separate microwave generators is practical in "proof-of-principle" experiments requiring two or three frequencies, however, future experiments should focus on exploiting techniques used in software defined radio, where a multifrequency signal is synthesized using digital signal processing, before being up-converted to microwave frequencies using a mixer and a reference source [67].

\section{APPENDIX D: ELECTRON POPULATION}

Here we present the data used to determine the electron population of the DQD. In Fig. 12(a), we enlarge a charge transition of the $B 1$ dot and count the discontinuities, which indicate loading of electrons into the $T 1 \mathrm{dot}$, marked by the white dashed lines. No discontinuities are observed at $V_{T 1}<0.375 \mathrm{~V}$, so we conclude that the first 

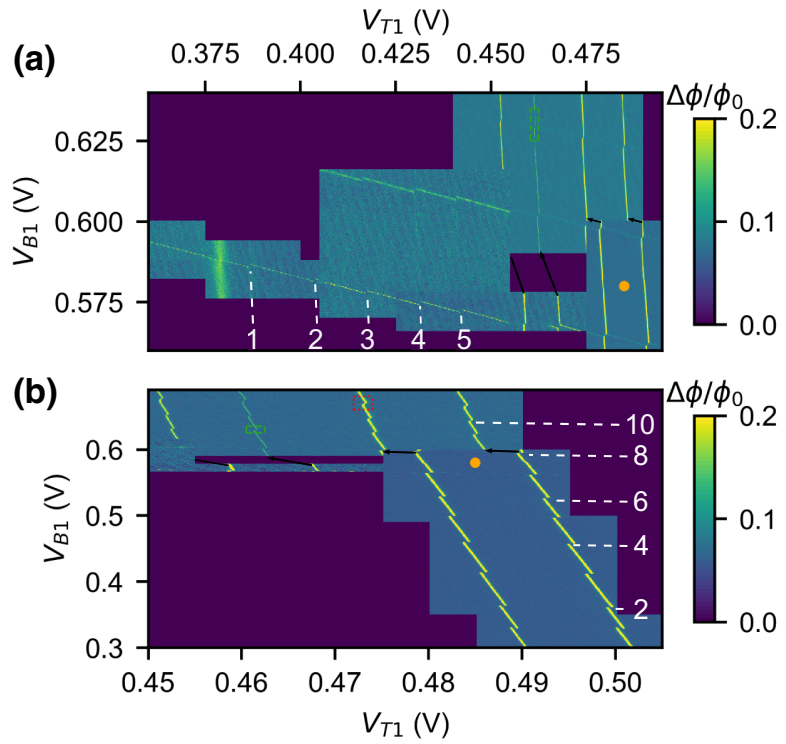

FIG. 12. Determining the electron occupancy. (a) Charge stability plot focusing on a charge transition of the $B 1$ dot, where we indicate loading of electrons into the $T 1$ dot with the white dashed lines. (b) Charge stability plot showing a transition of the $T 1$ dot where we indicate loading of electrons into the $B 1$ dot. The red-dotted and green-dashed boxes indicate the ICTs investigated in Figs. 2 and 4 respectively, similar to those in Fig. 1(d). An orange marker is included in both plots at the same voltage coordinates $\left(V_{T 1}, V_{B 1}\right)=(0.485,0.58) \mathrm{V}$ to aid comparison. Due to an extended time period between some of the measurements, there is a variation in the position of 71 -dot charge transitions due to voltage drift; black arrows are used to connect these transitions between the data sets.

shown here, occurring at $V_{T 1} \approx 0.387 \mathrm{~V}$, corresponds to the first electron loaded into the $T 1$ dot. In Fig. 12(b), we follow a transition of the $T 1$ dot and count electrons loading into the $B 1$ dot. No more discontinuities of this $T 1$ transition are observed for $V_{B 1}<0.3 \mathrm{~V}$. The ICT investigated in Figs. 2 and 4 is indicated by the red dotted (green dashed) box, similarly to in Fig. 1(d).

\section{APPENDIX E: LARGE DISPERSIVE SHIFT WITH A DIFFERENT NWFET}

In this section, we show data obtained using our resonator with a different NWFET device. We exchange the device measured in the main section of the paper for one with a metal super-top gate, which fully covers the poly-Si split gates, as shown in Fig. 13(a). The super-top gate is separated from the split-gates by $30 \mathrm{~nm}$ of $\mathrm{Si}_{3} \mathrm{~N}_{4}$ followed by a $350-\mathrm{nm}$ oxide stack. The nanowire and gate dimensions are the same as the previous device, but the devices are on different chips. The cavity resonance decreases slightly to $f_{0}=1.814 \mathrm{GHz}$, which may be caused by a combination of the larger area of this silicon chip, and a small increase in the bond-wire length. We tune the voltages of the three gates to the ICT shown in Fig. 13(b),

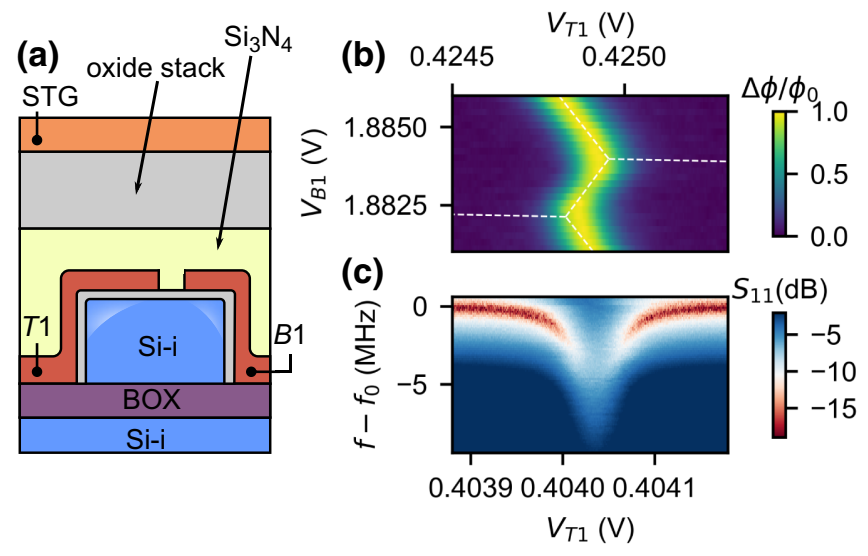

FIG. 13. Large dispersive interaction in a NWFET with a super-top gate (STG). (a) Cross-section schematic of the NWFET, similar to Fig. 1(b), but with an additional gate STG above the split gate. (b) Reduced phase response of the resonator $\Delta \phi / \phi_{0}$ in the region of an ICT. (c) Magnitude of the signal reflected from the cavity showing the shift in the resonant frequency as $V_{T 1}$ is swept across the ICT. Note the change in $V_{T 1}$ axes is due to a change in the $V_{\mathrm{STG}}$ setting: $V_{\mathrm{STG}}=11.15 \mathrm{~V}$ in (a) and $12.1 \mathrm{~V}$ in (b).

where we estimate the total electron occupancy of the DQD to be around 20, similar to the dispersive regime in the previous device. We observe a large dispersive shift of $3.6 \mathrm{MHz}$ in the cavity frequency, shown in Fig. 13(c), similar to the shift observed in Fig. 2(c) with the previous device. These results demonstrate the reproducibility of our approach across different devices.

\section{APPENDIX F: CONDITION OF MAXIMUM STATE VISIBILITY FOR SINGLET-TRIPLET READOUT}

In order to calculate the condition of maximum state visibility, we calculate the maximum change in reflection coefficient between a singlet outcome, which induces a frequency shift $\chi$, and triplet outcome that does not shift the resonator frequency [38]. Using Eq. (2), we find

$$
\begin{aligned}
& \Gamma_{s}=1+\frac{i \kappa_{\mathrm{ext}}}{\Delta_{0}-\chi-i \kappa / 2}, \\
& \Gamma_{t}=1+\frac{i \kappa_{\mathrm{ext}}}{\Delta_{0}-i \kappa / 2} .
\end{aligned}
$$

We calculate the change in reflection coefficient $\Delta \Gamma=$ $\Gamma_{s}-\Gamma_{t}$ and find

$$
\Delta \Gamma=i \frac{\kappa_{\mathrm{ext}} \chi}{\left(\Delta_{0}^{2}-\Delta_{0} \chi-\kappa^{2} / 4\right)-i \kappa\left(\Delta_{0}-\chi / 2\right)} .
$$

The first condition for maximum state visibility occurs when $\operatorname{Re}(\Delta \Gamma)=0$. This requirement set the optimal operation detuning from the bare cavity frequency at 
$\Delta_{0}=\chi / 2$, i.e., at the average frequency between singlet and triplet outcomes. In this case,

$$
\Delta \Gamma=\frac{i 4 \kappa_{\mathrm{ext}} \chi}{\chi^{2}+\kappa^{2}} .
$$

Equation (F3) is maximum when $\chi=\kappa$, the condition of maximum state visibility. At this point $\Delta \Gamma=$ $i(2 \beta / 1+\beta)$, where $\beta=\kappa_{\text {ext }} / \kappa_{\text {int }}$ is the coupling coefficient. Finally, we calculate the SNR, knowing that photons leak out of the resonator at a rate $\kappa_{\text {ext }}$

$$
\mathrm{SNR}=|\Delta \Gamma|^{2} \frac{\bar{n}}{n_{\text {noise }}} \frac{\kappa_{\mathrm{ext}}}{2 \pi} t_{\text {int }},
$$

where $n_{\text {noise }}=k_{B} T_{N} / h f_{0}$ is the photon noise and $\bar{n}$ is the number of photons in the resonator. At the condition for maximum state visibility, for a critically coupled resonator driven at the critical photon number $\bar{n}=n_{\text {crit }}=\Delta^{2} /\left(4 g_{0}^{2}\right)$, we find

$$
\mathrm{SNR}=\frac{h f_{0}}{k_{B} T_{N}} \frac{g_{0}^{2}}{\kappa} t_{\mathrm{int}}
$$

[1] Floris A. Zwanenburg, Andrew S. Dzurak, Andrea Morello, Michelle Y. Simmons, Lloyd C. L. Hollenberg, Gerhard Klimeck, Sven Rogge, Susan N. Coppersmith, and Mark A. Eriksson, Silicon quantum electronics, Rev. Mod. Phys. 85, 961 (2013).

[2] Jun Yoneda, Kenta Takeda, Tomohiro Otsuka, Takashi Nakajima, Matthieu R. Delbecq, Giles Allison, Takumu Honda, Tetsuo Kodera, Shunri Oda, and Yusuke Hoshi et al., A quantum-dot spin qubit with coherence limited by charge noise and fidelity higher than $99.9 \%$, Nat. Nanotechnol. 13, 102 (2018).

[3] C. H. Yang, K. W. Chan, R. Harper, W. Huang, T. Evans, J. C. C. Hwang, B. Hensen, A. Laucht, T. Tanttu, F. E. Hudson, S. T. Flammia, K. M. Itoh, A. Morello, S. D. Bartlett, and A. S. Dzurak, Silicon qubit fidelities approaching incoherent noise limits via pulse engineering, Nat. Electron. 2, 151 (2019).

[4] W. Huang, C. H. Yang, K. W. Chan, T. Tanttu, B. Hensen, R. C. C. Leon, M. A. Fogarty, J. C. C. Hwang, F. E. Hudson, K. M. Itoh, A. Morello, A. Laucht, and A. S. Dzurak, Fidelity benchmarks for two-qubit gates in silicon, Nature 569, 532 (2019).

[5] X. Xue, T. F. Watson, J. Helsen, D. R. Ward, D. E. Savage, M. G. Lagally, S. N. Coppersmith, M. A. Eriksson, S. Wehner, and L. M. K. Vandersypen, Benchmarking Gate Fidelities in a Si/SiGe Two-Qubit Device, Phys. Rev. X 9, 021011 (2019).

[6] David M. Zajac, Anthony J. Sigillito, Maximilian Russ, Felix Borjans, Jacob M. Taylor, Guido Burkard, and Jason R. Petta, Resonantly driven CNOT gate for electron spins, Science 359, 439 (2018).

[7] Louis Hutin, B. Bertrand, Romain Maurand, A. Crippa, M. Urdampilleta, Y. J. Kim, A. Amisse, H. Bohuslavskyi, L.
Bourdet, and Sylvain Barraud et al., in 2018 48th European Solid-State Device Research Conference (ESSDERC) (IEEE, Dresden, 2018), p. 12.

[8] R. Pillarisetty, H. C. George, T. F. Watson, L. Lampert, N. Thomas, S. Bojarski, P. Amin, R. Caudillo, E. Henry, and N. Kashani et al., in 2019 IEEE International Electron Devices Meeting (IEDM) (IEEE, San Francisco, 2019), p. 31.

[9] A. M. J. Zwerver, T. Krähenmann, T. F. Watson, L. Lampert, H. C. George, R. Pillarisetty, S. A. Bojarski, P. Amin, S. V. Amitonov, and J. M. Boter et al., Qubits made by advanced semiconductor manufacturing, arXiv preprint arXiv:2101.12650 (2021).

[10] Simon Schaal, Alessandro Rossi, Virginia N. Ciriano-Tejel, Tsung-Yeh Yang, Sylvain Barraud, John J. L. Morton, and M. Fernando Gonzalez-Zalba, A CMOS dynamic random access architecture for radio-frequency readout of quantum devices, Nat. Electron. 2, 236 (2019).

[11] Austin G. Fowler, Matteo Mariantoni, John M. Martinis, and Andrew N. Cleland, Surface codes: Towards practical large-scale quantum computation, Phys. Rev. A 86, 032324 (2012).

[12] M. Veldhorst, J. C. C Hwang, C. H. Yang, A. W. Leenstra, Bob de Ronde, J. P. Dehollain, J. T. Muhonen, F. E. Hudson, Kohei M. Itoh, and A. Morello et al., An addressable quantum dot qubit with fault-tolerant control-fidelity, Nat. Nanotechnol. 9, 981 (2014).

[13] M. J. Curry, M. Rudolph, T. D. England, A. M. Mounce, R. M. Jock, C. Bureau-Oxton, P. Harvey-Collard, P. A. Sharma, J. M. Anderson, D. M. Campbell, J. R. Wendt, D. R. Ward, S. M. Carr, M. P. Lilly, and M. S. Carroll, Singleshot readout performance of two heterojunction-bipolartransistor amplification circuits at millikelvin temperatures, Sci. Rep. 9, 16976 (2019).

[14] Matias Urdampilleta, David J. Niegemann, Emmanuel Chanrion, Baptiste Jadot, Cameron Spence, Pierre-André Mortemousque, Christopher Bäuerle, Louis Hutin, Benoit Bertrand, Sylvain Barraud, Romain Maurand, Marc Sanquer, Xavier Jehl, Silvano De Franceschi, Maud Vinet, and Tristan Meunier, Gate-based high fidelity spin readout in a CMOS device, Nat. Nanotechnol. 14, 737 (2019).

[15] Christian Volk, Anasua Chatterjee, Fabio Ansaloni, Charles M. Marcus, and Ferdinand Kuemmeth, Fast charge sensing of $\mathrm{Si} / \mathrm{SiGe}$ quantum dots via a high-frequency accumulation gate, Nano Lett. 19, 5628 (2019).

[16] D. Keith, M. G. House, M. B. Donnelly, T. F. Watson, B. Weber, and M. Y. Simmons, Single-Shot Spin Readout in Semiconductors near the Shot-Noise Sensitivity Limit, Phys. Rev. X 9, 041003 (2019).

[17] Alexandre Blais, Ren-Shou Huang, Andreas Wallraff, S. M. Girvin, and R. J. Schoelkopf, Cavity quantum electrodynamics for superconducting electrical circuits: An architecture for quantum computation, Phys. Rev. A 69, 062320 (2004).

[18] Andreas Wallraff, David I. Schuster, Alexandre Blais, Luigi Frunzio, R.-S. Huang, Johannes Majer, Sameer Kumar, Steven M. Girvin, and Robert J. Schoelkopf, Strong coupling of a single photon to a superconducting qubit using circuit quantum electrodynamics, Nature 431, 162 (2004). 
[19] Alexandre Blais, Arne L. Grimsmo, S. M. Girvin, and Andreas Wallraff, Circuit quantum electrodynamics, Rev. Mod. Phys. (2020).

[20] Anderson West, Bas Hensen, Alexis Jouan, Tuomo Tanttu, Chih-Hwan Yang, Alessandro Rossi, M. Fernando Gonzalez-Zalba, Fay Hudson, Andrea Morello, and David J. Reilly et al., Gate-based single-shot readout of spins in silicon, Nat. Nanotechnol. 14, 437 (2019).

[21] P. Pakkiam, A. V. Timofeev, M. G. House, M. R. Hogg, T. Kobayashi, M. Koch, S. Rogge, and M. Y. Simmons, Single-Shot Single-Gate rf Spin Readout in Silicon, Phys. Rev. X 8, 041032 (2018).

[22] Guoji Zheng, Nodar Samkharadze, Marc L. Noordam, Nima Kalhor, Delphine Brousse, Amir Sammak, Giordano Scappucci, and Lieven M. K. Vandersypen, Rapid gatebased spin read-out in silicon using an on-chip resonator, Nat. Nanotechnol. 14, 742 (2019).

[23] Nodar Samkharadze, Guoji Zheng, Nima Kalhor, Delphine Brousse, Amir Sammak, U. C. Mendes, Alexandre Blais, Giordano Scappucci, and L. M. K Vandersypen, Strong spin-photon coupling in silicon, Science 359, 1123 (2018).

[24] Anna Stockklauser, Pasquale Scarlino, J. V. Koski, Simone Gasparinetti, C. K. Andersen, Christian Reichl, Werner Wegscheider, Thomas Ihn, Klaus Ensslin, and Andreas Wallraff, Strong Coupling Cavity qed with Gate-Defined Double Quantum Dots Enabled by a High Impedance Resonator, Phys. Rev. X 7, 011030 (2017).

[25] M. Veldhorst, H. G. J. Eenink, C. H. Yang, and A. S. Dzurak, Silicon cmos architecture for a spin-based quantum computer, Nat. Commun. 8, 1766 (2017).

[26] Ruoyu Li, Luca Petit, David P. Franke, Juan Pablo Dehollain, Jonas Helsen, Mark Steudtner, Nicole K. Thomas, Zachary R. Yoscovits, Kanwal J. Singh, Stephanie Wehner, Lieven M. K. Vandersypen, James S. Clarke, and Menno Veldhorst, A crossbar network for silicon quantum dot qubits, Sci. Adv. 4, eaar3960 (2018).

[27] David Isaac Schuster, Ph.D. thesis, Department of Physics, Yale University, New Haven (2007).

[28] Virginia N. Ciriano-Tejel, Michael A. Fogarty, Simon Schaal, Louis Hutin, Benoit Bertrand, M. Fernando Gonzalez-Zalba, Jing Li, Y-M. Niquet, Maud Vinet, and John J. L. Morton, Spin Readout of a Cmos Quantum Dot by Gate Reflectometry and Spin-Dependent Tunnelling, PRX Quantum 2, 010353 (2021).

[29] Xiao Mi, Mónica Benito, Stefan Putz, David M. Zajac, Jacob M. Taylor, Guido Burkard, and Jason R. Petta, A coherent spin-photon interface in silicon, Nature 555, 599 (2018).

[30] Damaz de Jong, Jasper Van Veen, Luca Binci, Amrita Singh, Peter Krogstrup, L. P. Kouwenhoven, Wolfgang Pfaff, and J. D. Watson, Rapid Detection of Coherent Tunneling in an Inas Nanowire Quantum dot through Dispersive Gate Sensing, Phys. Rev. Appl. 11, 044061 (2019).

[31] Deividas Sabonis, Eoin C. T. O'Farrell, Davydas Razmadze, David M. T. van Zanten, Judith Suter, Peter Krogstrup, and Charles M. Marcus, Dispersive sensing in hybrid inas/al nanowires, Appl. Phys. Lett. 115, 102601 (2019).

[32] Theodor Lundberg, Jing Li, Louis Hutin, Benoit Bertrand, David J. Ibberson, Chang-Min Lee, David J. Niegemann,
Matias Urdampilleta, Nadia Stelmashenko, and Tristan Meunier et al., A Spin Quintet in a Silicon Double Quantum Dot: Spin Blockade and Relaxation, Phys. Rev. X 10, 041010 (2020).

[33] A. C. Betz, Romain Wacquez, Maud Vinet, Xavier Jehl, A. L. Saraiva, M. Sanquer, A. J. Ferguson, and M. F. Gonzalez-Zalba, Dispersively detected pauli spin-blockade in a silicon nanowire field-effect transistor, Nano Lett. 15, 4622 (2015).

[34] David S. Wisbey, Jiansong Gao, Michael R. Vissers, Fabio C. S. da Silva, Jeffrey S. Kline, Leila Vale, and David P. Pappas, Effect of metal/substrate interfaces on radiofrequency loss in superconducting coplanar waveguides, J. Appl. Phys. 108, 093918 (2010).

[35] M. S. Khalil, M. J. A Stoutimore, F. C. Wellstood, and K. D. Osborn, An analysis method for asymmetric resonator transmission applied to superconducting devices, J. Appl. Phys. 111, 054510 (2012).

[36] Imtiaz Ahmed, J. A. Haigh, Simon Schaal, Sylvain Barraud, Yi Zhu, Chang-min Lee, Mario Amado, J. W. A. Robinson, Alessandro Rossi, J. J. L. Morton, and M. F. Gonzalez-Zalba, Radio-Frequency Capacitive Gate-Based Sensing, Phys. Rev. Appl. 10, 014018 (2018).

[37] David J. Ibberson, Lisa A. Ibberson, Geoff Smithson, James A. Haigh, Sylvain Barraud, and M. Fernando GonzalezZalba, Low-temperature tunable radio-frequency resonator for sensitive dispersive readout of nanoelectronic devices, Appl. Phys. Lett. 114, 123501 (2019).

[38] R. Mizuta, R. M. Otxoa, A. C. Betz, and M. F. GonzalezZalba, Quantum and tunneling capacitance in charge and spin qubits, Phys. Rev. B 95, 045414 (2017).

[39] Monica Benito, Xiao Mi, Jacob M. Taylor, Jason R. Petta, and Guido Burkard, Input-output theory for spin-photon coupling in si double quantum dots, Phys. Rev. B 96, 235434 (2017).

[40] Will Gilbert, Andre Saraiva, Wee Han Lim, Chih Hwan Yang, Arne Laucht, Benoit Bertrand, Nils Rambal, Louis Hutin, Christopher C. Escott, and Maud Vinet et al., Singleelectron operation of a silicon-cmos $2 \times 2$ quantum dot array with integrated charge sensing, Nano Lett. 20, 7882 (2020).

[41] C. H. Yang, A. Rossi, R. Ruskov, N. S. Lai, F. A. Mohiyaddin, S. Lee, C. Tahan, Gerhard Klimeck, A. Morello, and A. S. Dzurak, Spin-valley lifetimes in a silicon quantum dot with tunable valley splitting, Nat. Commun. 4, 2069 (2013).

[42] L. Childress, A. S. Sørensen, and Mikhail D. Lukin, Mesoscopic cavity quantum electrodynamics with quantum dots, Phys. Rev. A 69, 042302 (2004).

[43] Karl D. Petersson, Louis W. McFaul, Michael D. Schroer, Minkyung Jung, Jacob M. Taylor, Andrew A. Houck, and Jason R. Petta, Circuit quantum electrodynamics with a spin qubit, Nature 490, 380 (2012).

[44] E. Dupont-Ferrier, B. Roche, B. Voisin, X. Jehl, R. Wacquez, M. Vinet, M. Sanquer, and S. De Franceschi, Coherent Coupling of two Dopants in a Silicon Nanowire Probed by Landau-Zener-Stückelberg Interferometry, Phys. Rev. Lett. 110, 136802 (2013).

[45] M. Fernando Gonzalez-Zalba, Sergey N. Shevchenko, Sylvain Barraud, J. Robert Johansson, Andrew J. Ferguson, Franco Nori, and Andreas C. Betz, Gate-sensing coherent 
charge oscillations in a silicon field-effect transistor, Nano Lett. 16, 1614 (2016).

[46] Anasua Chatterjee, S. N. Shevchenko, Sylvain Barraud, R. M. Otxoa, Franco Nori, J. J. L. Morton, and M. F. Gonzalez-Zalba, A silicon-based single-electron interferometer coupled to a fermionic sea, Phys. Rev. B 97, 045405 (2018).

[47] Alexandre Blais, Ren-Shou Huang, Andreas Wallraff, S. M. Girvin, and R. J. Schoelkopf, Cavity quantum electrodynamics for superconducting electrical circuits: An architecture for quantum computation, Phys. Rev. A 69, 062320 (2004).

[48] Fei Yan, Simon Gustavsson, Archana Kamal, Jeffrey Birenbaum, Adam P. Sears, David Hover, Ted J. Gudmundsen, Danna Rosenberg, Gabriel Samach, and Steven Weber et al., The flux qubit revisited to enhance coherence and reproducibility, Nat. Commun. 7, 12964 (2016).

[49] Vahid Derakhshan Maman, M. F. Gonzalez-Zalba, and András Pályi, Charge Noise and Overdrive Errors in Reflectometry-Based Charge, Spin and Majorana Qubit Readout, Phys. Rev. Applied 14, 064024 (2020).

[50] S. Schaal, I. Ahmed, J. A. Haigh, L. Hutin, B. Bertrand, S. Barraud, M. Vinet, C-M. Lee, N. Stelmashenko, J. W. A. Robinson, J. Y. Qiu, S. Hacohen-Gourgy, I. Siddiqi, M. F. Gonzalez-Zalba, and J. J. L. Morton, Fast GateBased Readout of Silicon Quantum Dots Using Josephson Parametric Amplification, Phys. Rev. Lett. 124, 067701 (2020).

[51] J. Stehlik, Y-Y Liu, C. M. Quintana, C. Eichler, T. R. Hartke, and J. R. Petta, Fast Charge Sensing of a CavityCoupled Double Quantum dot Using a Josephson Parametric Amplifier, Phys. Rev. Appl. 4, 014018 (2015).

[52] Christian Barthel, D. J. Reilly, C. M. Marcus, M. P. Hanson, and A. C. Gossard, Rapid Single-Shot Measurement of a Singlet-Triplet Qubit, Phys. Rev. Lett. 103, 160503 (2009).

[53] J. M. Hornibrook, J. I. Colless, A. C. Mahoney, X. G. Croot, S. Blanvillain, H. Lu, A. C. Gossard, and D. J. Reilly, Frequency multiplexing for readout of spin qubits, Appl. Phys. Lett. 104, 103108 (2014).

[54] Johannes Heinsoo, C. K. Andersen, Ants Remm, Sebastian Krinner, Theodore Walter, Yves Salathé, Simone Gasparinetti, Jean-Claude Besse, Anton Potočnik, Andreas Wallraff, and Christopher Eichler, Rapid High-Fidelity Multiplexed Readout of Superconducting Qubits, Phys. Rev. Appl. 10, 034040 (2018).

[55] Jens Koch, T. M. Yu, Jay Gambetta, A. A. Houck, D. I. Schuster, J. Majer, Alexandre Blais, M. H. Devoret, S. M. Girvin, and R. J. Schoelkopf, Charge-insensitive qubit design derived from the cooper pair box, Phys. Rev. A 76, 042319 (2007).

[56] Péter Boross, Gábor Széchenyi, and András Pályi, Valleyenhanced fast relaxation of gate-controlled donor qubits in silicon, Nanotechnology 27, 314002 (2016).

[57] Xiao Mi, J. V. Cady, D. M. Zajac, P. W. Deelman, and Jason R. Petta, Strong coupling of a single electron in silicon to a microwave photon, Science 355, 156 (2017).

[58] Pasquale Scarlino, D. J. van Woerkom, Anna Stockklauser, J. V. Koski, M. C. Collodo, Simone Gasparinetti, Christian Reichl, Werner Wegscheider, Thomas Ihn, Klaus Ensslin, and Andreas Wallraff, All-Microwave Control and Dispersive Readout of Gate-Defined Quantum Dot Qubits in Circuit Quantum Electrodynamics, Phys. Rev. Lett. 122, 206802 (2019).

[59] A. Crippa, R. Ezzouch, A. Aprá, A. Amisse, R. Laviéville, L. Hutin, B. Bertrand, M. Vinet, M. Urdampilleta, and T. Meunier et al., Gate-reflectometry dispersive readout and coherent control of a spin qubit in silicon, Nat. Commun. 10, 1 (2019).

[60] R. Maurand, X. Jehl, D. Kotekar-Patil, A. Corna, H. Bohuslavskyi, R. Laviéville, L. Hutin, S. Barraud, M. Vinet, and M. Sanquer et al., A CMOS silicon spin qubit, Nat. Commun. 7, 1 (2016).

[61] P. Forn-Díaz, L. Lamata, E. Rico, J. Kono, and E. Solano, Ultrastrong coupling regimes of light-matter interaction, Rev. Mod. Phys. 91, 025005 (2019).

[62] http://mos-quito.eu.

[63] Moe S. Khalil, F. C. Wellstood, and Kevin D. Osborn, Loss dependence on geometry and applied power in superconducting coplanar resonators, IEEE Trans. Appl. Supercond. 21, 879 (2010).

[64] Jeremy M. Sage, Vladimir Bolkhovsky, William D. Oliver, Benjamin Turek, and Paul B. Welander, Study of loss in superconducting coplanar waveguide resonators, J. Appl. Phys. 109, 063915 (2011).

[65] K. Hayashi, A. Saito, Y. Ogawa, M. Murata, T. Sawada, K. Nakajima, H. Yamada, S. Ariyoshi, T. Taino, and H. Tanoue et al., in 11th European Conference on Applied Superconductivity (EUCAS2013) (IOP Publishing, Genoa, Italy, 2014), Vol. 507, p. 042015.

[66] M. Peruzzo, A. Trioni, F. Hassani, M. Zemlicka, and J. M. Fink, Surpassing the Resistance Quantum with a Geometric Superinductor, Phys. Rev. Appl. 14, 044055 (2020).

[67] Markus Jerger, Stefano Poletto, Pascal Macha, Uwe Hübner, Evgeni Il'ichev, and Alexey V. Ustinov, Frequency division multiplexing readout and simultaneous manipulation of an array of flux qubits, Appl. Phys. Lett. 101, 042604 (2012). 